\title{
Ibutilide Fumarate
}

National Cancer Institute

\section{Source}

National Cancer Institute. Ibutilide Fumarate. NCI Thesaurus. Code C47561.

The fumarate salt form of ibutilide, a class III antiarrhythmic agent. Ibutilide exerts its effect by activating a slow, inward, predominately sodium current rather than by blocking outward potassium currents. This results in prolong ation of atrial and ventricular action potential duration and refractory periods. Ibutilide slightly decreases the sinus rate and atrioventricular (AV) conduction and produces a dose-related prolongation of the QT interval. ( $\mathrm{NCl} 05)$ 